\title{
Perfil sociodemográfico de los commuters en las zonas metropolitanas del estado de Hidalgo, 2015
}

\author{
*Omar Villanueva-Mejía \\ *Laura Myriam Franco-Sánchez \\ **Sergio Cuauhtémoc Gaxiola Robles-Linares \\ *Universidad Autónoma del Estado de Hidalgo, México \\ **Universidad Autónoma del Estado de México, México
}

\section{Resumen}

Las zonas metropolitanas de México se constituyen como espacios urbanos con complejos procesos en su dinámica interior y en su interacción con las áreas circundantes. Donde los flujos constantes de personas que se trasladan a realizar sus actividades diarias fuera de su lugar de residencia hace de la movilidad cotidiana un elemento relevante en el estudio de las ciudades. Este artículo se centra en el problema de la movilidad cotidiana al lugar de trabajo en el estado de Hidalgo para el año 2015. En el documento, se presentan los principales elementos conceptuales que delimitan el estudio de la movilidad cotidiana al trabajo y su relación con el término commuting, además de adentrarse en la contextualización del problema de la movilidad cotidiana en las ciudades, centrándose en el resultado de la aplicación de una regresión logística binaria, también se presenta el perfil sociodemográfico de la población que trabaja en un municipio diferente al que reside. El objetivo es caracterizar sociodemográficamente a las personas del estado de Hidalgo que en el año 2015 presentaron movilidad cotidiana al trabajo, esto permitirá saber y entender quiénes se mueven, qué escolaridad tienen, cuál es su situación conyugal, si son migrantes recientes, en qué sectores de la economía se emplean y la posición que ocupan en el trabajo.

Palabras clave: Movilidad cotidiana, commuters, perfil sociodemográfico.

\section{Sociodemographic characteristics of the commuters in the metropolitan zones} of the State of Hidalgo in 2015

\begin{abstract}
The metropolitan areas of Mexico are constituted as urban spaces with complex processes in their internal dynamics and their interaction with the surrounding areas, the constant flows of people who move to carry out their daily activities outside their place of residence makes daily mobility a relevant element in the study of cities. This article focuses on the problem of daily mobility to work in the State of Hidalgo for 2015 , presenting the main conceptual elements that define the study of daily mobility to work and its relationship with the term commuting, in addition to entering In the contextualisation of the problem of daily mobility in cities, focusing on the result of the application of a binary logistic regression, we present the sociodemographic profile of the population working in a different municipality to where they reside, the objective is to characterize people sociodemographically of the state of Hidalgo that for 2015 present daily mobility to work, this will allow to know and understand who move, what schooling they have, what their conjugal situation is, if they are recent migrants, in what sectors of the economy they are employed or the position they Occupy at work.
\end{abstract}

Key words: Daily mobility, commuters, sociodemographic profile.

Recibido: 26 de junio de 2018

Aprobado: 30 de abril de 2019 


\section{INTRODUCCIÓN}

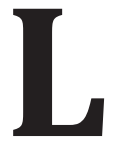

a fuerte reestructuración de las áreas urbanas y rurales, ha transformado las relaciones funcionales entre las diferentes zonas metropolitanas, es inminente el flujo y el intercambio de bienes, servicios, y por supuesto de población, que al relocalizarse las actividades económicas y transformar la dinámica de las ciudades se enfrenta a procesos como cambios de residencia por falta de acceso a infraestructura o altos costos del suelo que encarece el acceso a la vivienda en los lugares de origen. Esto ocasiona que la población busque alternativas asumiendo los costos de un cambio de residencia, ya que al moverse a otras ciudades de mayor tamaño y con infraestructura creciente aseguran educación, servicios de salud, servicios públicos de calidad, mayor seguridad; pero asumen en gran medida el hecho que tendrán que buscar nuevamente un empleo o bien trasladarse al trabajo que ya tenían. Es así como la mayoría de los desplazamientos actuales se realizan en busca de un empleo asalariado (Recio et al. 2006: 173), en general bajo la promesa de un mejor nivel de vida en la ciudad de destino. Esto genera la necesidad de moverse hacia los puntos donde se llevan a cabo las actividades cotidianas, y por ende el retorno al lugar de residencia al concluir dichas actividades (Susino y Martínez, 2009). La finalidad de este trabajo se centra en analizar el perfil sociodemográfico de las personas que hacen estos traslados que implican el retorno diario o constante al lugar de residencia que será englobado en el término commuting o movilidad cotidiana. ${ }^{1}$

El estudio se realizará en las tres Zonas Metropolitanas de Hidalgo $(\mathrm{ZMH})$, y los sujetos de estudio serán las personas que trabajan en un municipio diferente al municipio donde residen. De esta forma, se tienen detectados de acuerdo a los micro datos de la Encuesta Intercensal 2015, que existen 107 mil 787 personas que presentan esta condición, de los cuales nos interesa conocer características como sexo, edad, escolaridad, situación conyugal, ocupación, el lugar al que

1 Movilidad cotidiana se refiere a los desplazamientos cortos, diarios y cíclicos entre un lugar de origen y uno de destino, ya sea en la misma unidad administrativa o en otra, nacional o de otro país. 
se traslada entre otras características. Por lo tanto, el resultado que se persigue es tener un perfil definido de estos sujetos.

\section{EL PROBLEMA DE LA MOVILIDAD: REVISIÓN CONCEPTUAL}

Las ciudades son un espacio natural para el estudio de la dinámica y los resultados de diversas pautas de movilidad humana. Desde la perspectiva de la ciudad el interés se ha centrado en las razones de la movilidad de las personas y de cómo trabajan, viven y configuran sus habitantes locales (OIM, 2015: 30).

La movilidad cotidiana como hecho social o acción social se entiende como trasladarse de un lugar a otro, implicando dos hechos subsecuentes, el primero, el salir de un lugar y el segundo implica llegar a otro lugar (Jiménez, 2008: 5). Si bien las causas de este tipo de movilidad pueden ser diversas, las dos principales son el trabajo y los estudios. El ritmo de crecimiento de la movilidad cotidiana de las personas al relacionarse con el mercado de trabajo es cada vez más diverso y complejo, y se expresa asociado a factores sociales y económicos, tales como la oferta de trabajo y el tipo de actividad económica prevaleciente en las diversas zonas del país (agropecuaria, minera, industrial, de comercio y/o servicios), las condiciones de desempleo y pobreza, la búsqueda de oportunidades de trabajo, entre otros (INEGI, 2015).

La movilidad cotidiana se identifica así con las prácticas habituales y reiteradas de desplazamientos de corta duración y distancia vinculados a distintos fines, donde predominan los desplazamientos al trabajo y al lugar de estudio, ${ }^{2}$ aunque existen otras motivaciones para esta movilidad como puede ser: ir de compras, ocio, ir a comer, visitar familiares y amigos, llevar o recoger a alguien, realizar trámites o acceder a servicios médicos (Casado, 2008).

La movilidad cotidiana involucra un desplazamiento entre dos lugares, con cierta regularidad o frecuencia, esto puede ser diario, varios días, o incluso una semana o más, caracterizándose como movilidad cotidiana al hecho de que siempre está presente el retorno al lugar de residencia. Dentro de la literatura sobre el tema será recurrente encontrar a esta movilidad cotidiana también referida como commute, la cual sería definida como viajes con cierta regularidad entre dos lugares como un suburbio y la ciudad (Jiménez, 2008).

2 Para Casado (2008) los desplazamientos al lugar de trabajo y al lugar de estudio en ocasiones se engloban como movilidad obligada. 
Al hablar de movilidad cotidiana entran en consideración las dimensiones espaciales, temporales y sociales las cuales presentan ambigüedades como la delimitación geográfica, la duración del traslado o el cambio de entorno social. Al poner en contexto estas dimensiones aproximan algo más a la idea diferenciada de lo que es un movimiento migratorio y lo que se define como movilidad cotidiana que es lo que nos atañe en este trabajo. En este sentido, la ambigüedad se refleja en el hecho de que un traslado desde las ZMH a otro estado o a la Ciudad de México durante toda la semana laboral, estaría considerado como commuting al igual que un traslado entre municipios de la misma zona metropolitana (ZM's) donde se retorne a casa el mismo día, esto para el caso que se tomará como unidad administrativa al municipio.

Además de las dimensiones espaciales, temporales y sociales, cuya relatividad es determinante en la línea que divide a la migración ${ }^{3}$ de la movilidad cotidiana, es imperante la presencia del "cambio permanente de residencia” para referirnos a la migración, que no necesariamente estaría contemplado al definir la movilidad o al menos no vista desde un "cambio permanente", sino solamente de un "cambio de residencia", quizá solo durante la semana laboral o el tiempo que la persona pretenda permanecer en este lugar. Aquí cabe discutir de entrada la dimensión espacial, ya que desde una visión economicista neoclásica habría una distancia donde el costo-beneficio de trasladarse llevaría a la decisión de permanecer ahí un día, una semana o más dependiendo del interés del individuo.

La movilidad cotidiana está acotada en primera instancia por la distancia entre el lugar de origen y el de destino o bien la complejidad para llegar o salir de este. Lo anterior conlleva a determinar el tiempo ${ }^{4}$ que se decida permanecer en el lugar de destino antes de retornar y con qué periodicidad se hará esta movilidad.

En lo que respecta a la dimensión social, en la movilidad cotidiana definitivamente está presente una modificación del entorno social y cultural, pero además las personas no pierden interacción en los lugares de origen y destino, aun cuando sus roles dentro de estos

3 Es necesario recalcar que este trabajo se centra en la movilidad cotidiana y no de migración.

4 La decisión de cada persona de optar por la movilidad cotidiana está vinculado a las características del lugar de origen y llegada, en cuanto a servicios se refiere. Habrá lugares más distantes donde la conectividad y el transporte permita retornos constantes, o bien donde la distancia sea relativamente corta pero implique cruce de límites administrativos y por tanto procedimientos administrativos que lleven a la decisión de retrasar el retorno al lugar de origen, lo anterior incluye la dimensión tanto espacial como temporal. 
lugares presenten cambios. De esta forma, en la dimensión social la movilidad sería entendida como la interrupción de actividades en un lugar y su reorganización en otro, proceso que estará presente de forma cíclica.

Así, sumando el análisis de estas dimensiones se llega a formular la siguiente definición de movilidad cotidiana, se entiende como movilidad cotidiana a todos los desplazamientos en los que el cambio de residencia no está presente, debido a que el retorno a la residencia en el lugar de origen se lleva a cabo con cierta periodicidad, dicho desplazamiento implicaría el cruce de un límite administrativo, donde la interrupción de actividades en un lugar y su reorganización en otro está presente de forma cíclica.

\section{EL COMMUTING Y LA MOVILIDAD COTIDIANA}

El commuting se puede definir como el desplazamiento que se produce por la disociación entre lugar de trabajo y/o estudio y lugar de residencia. Es decir, el individuo cambia de lugar de residencia, sin cambiar el sitio de trabajo y/o estudio, y viceversa. También, es preciso mencionar que a la persona que realiza este tipo de movimiento suele denominarse commuter (García, 2010). Es necesario señalar que se utilizarán los conceptos de movilidad pendular o cotidiana como sinónimos y que para este trabajo entenderemos entonces al commuting como movilidad cotidiana.

La movilidad de las personas es una variable fundamental para entender cómo se organizan las ciudades, en sus interrelaciones y jerarquías, en el mundo de hoy, dicha movilidad estructura hasta tal punto su vida cotidiana que la ciudad se define por la movilidad de sus habitantes. En los países con sistemas estadísticos muy consolidados, el conocimiento de la movilidad cotidiana por motivos laborales ha sido utilizado para varios fines, entre ellos para el análisis de la estructura territorial (Susino y Martínez, 2009).

\section{EL PROBLEMA DE LA MOVILIDAD COTIDIANA EN LAS CIUDADES}

La ciudad reúne una población que se caracteriza por una determinada composición geográfica social y étnica, esta ciudad a su vez es un mecanismo para potenciar las interacciones humanas (Roncanyolo, 1988). Además se concibe como el espacio de asentamiento por excelencia de un gran número de personas que intervienen 
significativamente en su entorno dotándola de servicios especializados, infraestructuras de comunicación con una dinámica intensa en cuanto a sus relaciones interpersonales, densidades de ocupación de medias a altas, un desarrollo económico importante (principalmente en actividades de orden terciario y otras especializadas). El estudio de la movilidad cotidiana en la ciudad permite identificar las entidades, municipios o delegaciones expulsoras y receptoras de la fuerza de trabajo y de la oferta educativa; esto permite ver, desde un enfoque complementario al de la población residente, las necesidades de infraestructura y servicios de las ciudades. También posibilita identificar la conectividad entre diversas zonas geográficas, las relaciones de centralidad y dependencia entre lo local y lo regional, así como definir y actualizar las zonas metropolitanas del país. Además, la movilidad cotidiana afecta de manera significativa el ingreso en los hogares, debido a los gastos en transporte, gasolina o alimentación generados fuera de casa; por ello la importancia de identificar a esta población y los lugares dónde suceden estos fenómenos (INEGI, 2015). Más allá de las múltiples dimensiones a considerar dentro de la diversidad urbana, al momento de estudiar la movilidad por encima de la consideración del lugar de origen, es preciso tener en cuenta otras variables, como la condición migratoria y los concomitantes beneficios y restricciones de derechos, las experiencias en el mercado laboral, los perfiles de género y edad, las pautas de distribución espacial, así como las distintas respuestas de los encargados de la prestación de servicios y de los residentes, adaptadas a las condiciones locales en la zona (Ibidem). La movilidad, la transformación y el crecimiento de la ciudad así como la mutación de los sistemas de transporte se convirtieron en los principales factores de las dinámicas urbanas en estas ciudades. De esta manera el crecimiento de estas metrópolis y la funcionalidad de los distintos espacios urbanos son un elemento fundamental para plantear la problemática de la movilidad urbana como del acceso de los individuos a la ciudad y a sus recursos. La movilidad cotidiana y en este caso el commuting constituyen un tema de investigación emergente. Por lo tanto, el análisis de los patrones del commuting resulta útil cuando se analiza el desarrollo de redes urbanas, ya que los viajes al trabajo representan la mayor parte de los viajes diarios a escala inter e intraurbana (Boix y Trullén, 2012).

Profundizar en dichos flujos de movilidad, sus orígenes, causas, consecuencias, características por colectivos de interés, entre otros 
aspectos, permite arrojar mayor luz sobre el proceso de movilidad cotidiana en el espacio urbano, al lograr un mayor conocimiento de las pautas de atracción, especialización o diversificación de los territorios producto de los cambios en la jerarquía urbana.

El interés sobre la movilidad cotidiana viene dado por el crecimiento de las aglomeraciones metropolitanas y la dispersión de lo que se ha referido como los "espacios de la vida", es decir, las áreas que cubren toda la movilidad habitual que se realiza como parte de las actividades de la vida, y que se relacionan estrechamente con la manera en que funcionan las ciudades. La alta movilidad cotidiana en una zona determinada opera como característica definitoria de una área metropolitana (Jiménez, 2008).

Tanto la migración urbana como la movilidad urbana comprenden una diversidad de movimientos de población que se producen por una serie de razones y que tienen distinta duración. La mayoría de ellos abarca distancias cortas, dentro de las fronteras nacionales. Independientemente del carácter de esos movimientos, las personas se trasladan a las zonas urbanas, confiando en beneficiarse de la mayor disponibilidad de servicios y en tener acceso a una mejor infraestructura y oportunidades de generación de ingresos que les permitan contar con medios de subsistencia más estables. Cuando la movilidad cotidiana está bien gestionada puede inducir una dinámica social y económica que mejore la capacidad de los migrantes urbanos para hacer frente a conmociones y presiones. Sin embargo, los movimientos hacia las ciudades también entrañan riesgos sociales. La movilidad urbana no controlada puede contribuir a la escasez de infraestructura, vivienda y servicios, y agravar también los problemas financieros y las dificultades de los responsables de las instituciones locales. Cuando la capacidad de las autoridades y de los mercados urbanos es insuficiente para proporcionar servicios adecuados de empleo, agua y saneamiento, vivienda decente, transporte eficiente y atención de salud de buena calidad, algunos segmentos de la población urbana quedan marginados y en situación aún más vulnerable (OIM, 2015). En el contexto de las zonas metropolitanas del centro de México, las ZMH resaltan por su conectividad y amplia interacción con la región por lo que se considera un escenario idóneo para el análisis de la movilidad cotidiana.

Este artículo se centra en la movilidad cotidiana o commuting dentro de las en el estado de Hidalgo, en este sentido la ubicación de Hidalgo 
en el centro del país, su cercanía en la ciudad de México y la amplia conectividad y relación con la zona centro de México se resaltan como los principales elementos que dan pertinencia a esta investigación. La factibilidad de hacer viajes cotidianos y con reiterada frecuencia son propicios para que un sector de la población esté dispuesta a trabajar en un lugar distinto al de su residencia. Para entender qué perfil tiene esta población commuter es necesario conocer las características sociodemográficas de la población del estado, lo que hacemos en el apartado siguiente.

\section{CARACTERÍSTICAS SOCIODEMOGRÁFICAS EN EL ESTADO DE HIDALGO}

De acuerdo al Instituto Nacional de Estadística y Geografía (INEGI), para el año 2015 en Hidalgo vivían 2 millones 858 mil 359 personas, posicionando al estado en el lugar 17 a nivel nacional con un 2.4 por ciento del total de la población del país. Existiendo un mayor número de mujeres, con 52.1 por ciento, por un 47.9 por ciento de hombres, con un índice de masculinidad de 91.92 hombres por cada cien mujeres. En lo que respecta al territorio, representa el 1.06 por ciento de la superficie del país.

El 52 por ciento de la población habita en el ámbito urbano mientras que el 48 por ciento lo hace en el rural, ${ }^{5}$ los números a nivel nacional son del 78 y 22 por ciento respectivamente. A este respecto Granados y Franco (2017), señalan que aun cuando la población rural ha decrecido, no así el número de localidades rurales, que han ido en aumento derivado de un proceso de dispersión de la población. En Hidalgo en comparación con los estados de la Zona Centro de México $(\mathrm{ZCM})^{6}$ se tiene mayor proporción de habitantes en zonas rurales, a pesar de que cuenta con tres zonas metropolitanas completas y un municipio dentro de la Zona Metropolitana de la Ciudad de México. La existencia de 16 municipios metropolitanos podría sostener la idea de un estado más urbano, cosa que no sucede así. La explicación a la mayor proporción de habitantes en ámbitos rurales puede ser la concentración de las zonas metropolitanas en el sur del estado y su

5 El número de habitantes que tiene una población determina si ésta es rural o urbana. De acuerdo con el INEGI, una población se considera rural cuando tiene menos de 2 mil 500 habitantes, mientras que la urbana es aquella donde viven 2 mil 500 o más personas personas.

6 Al referirnos a la Zona Centro de México estaremos contemplando a los estados de México, Morelos, Tlaxcala, Puebla, Ciudad de México y Querétaro aun cuando este último en alguna literatura revisada no lo contempla como parte de la ZCM. 
lejanía con las zonas serranas, esto se puede observar más adelante en el Figura 1. Respecto a la marginación, de acuerdo con el Consejo Nacional de Evaluación de la Política de Desarrollo Social (CONEVAL), en su informe de pobreza y evaluación, sitúa a Hidalgo como un estado de alta marginación, ubica principalmente los municipios del norte del estado con mayor rezago.

En Hidalgo la escolaridad acumulada promedio es de 8.7 años que equivale a segundo año de secundaria. En este rubro, el dato estatal no lejano del panorama nacional de 9.1 años, equivalente al inicio del bachillerato, pero al analizar la situación al interior del estado puede cambiar un poco el panorama, ya que como ejemplo la capital del estado Pachuca para el 2010 tenía un grado promedio de escolaridad de 10.1 años, algo contrastante es que existen zonas al interior de la ciudad con niveles de escolaridad muy bajos, principalmente el rezago educativo se presenta en mujeres de colonias muy específicas donde el rezago es elevado (Villanueva, 2013).

En la parte económica, el sector de actividad que más aporta al Producto interno Bruto (PIB) estatal es el comercio, es decir, el 54 por ciento de la actividad se la lleva el sector terciario, y en general, el estado aporta 1.7 por ciento al PIB nacional. En el 2015 en el estado de Hidalgo había 137 personas por kilómetro cuadrado.

En lo concerniente a movimientos migratorios el estado de Hidalgo tiene fuertes flujos tanto internos como internacionales, donde de acuerdo al INEGI en el 2015, los principales estados con mayor intercambio migratorio con Hidalgo, son el Estado de México y la Ciudad de México. Mientras tanto, Estados Unidos de América es el principal destino de los migrantes internacionales con el 98 por ciento.

Una vez expuesto un panorama general de la situación sociodemográfica de Hidalgo, acotamos que nos centraremos en únicamente tres zonas metropolitanas. ${ }^{7}$ La población objetivo son las personas que presentan movilidad cotidiana al trabajo, y lo que se persigue es determinar mediante un perfil sociodemográfico el comportamiento de esta población en cuanto a movilidad cotidiana se refiere.

$\overline{7}$ No se contempla al municipio de Tizayuca que forma parte de la Zona Metropolitana de la Ciudad de México. 
Figura 1: Los municipios metropolitanos de Hidalgo
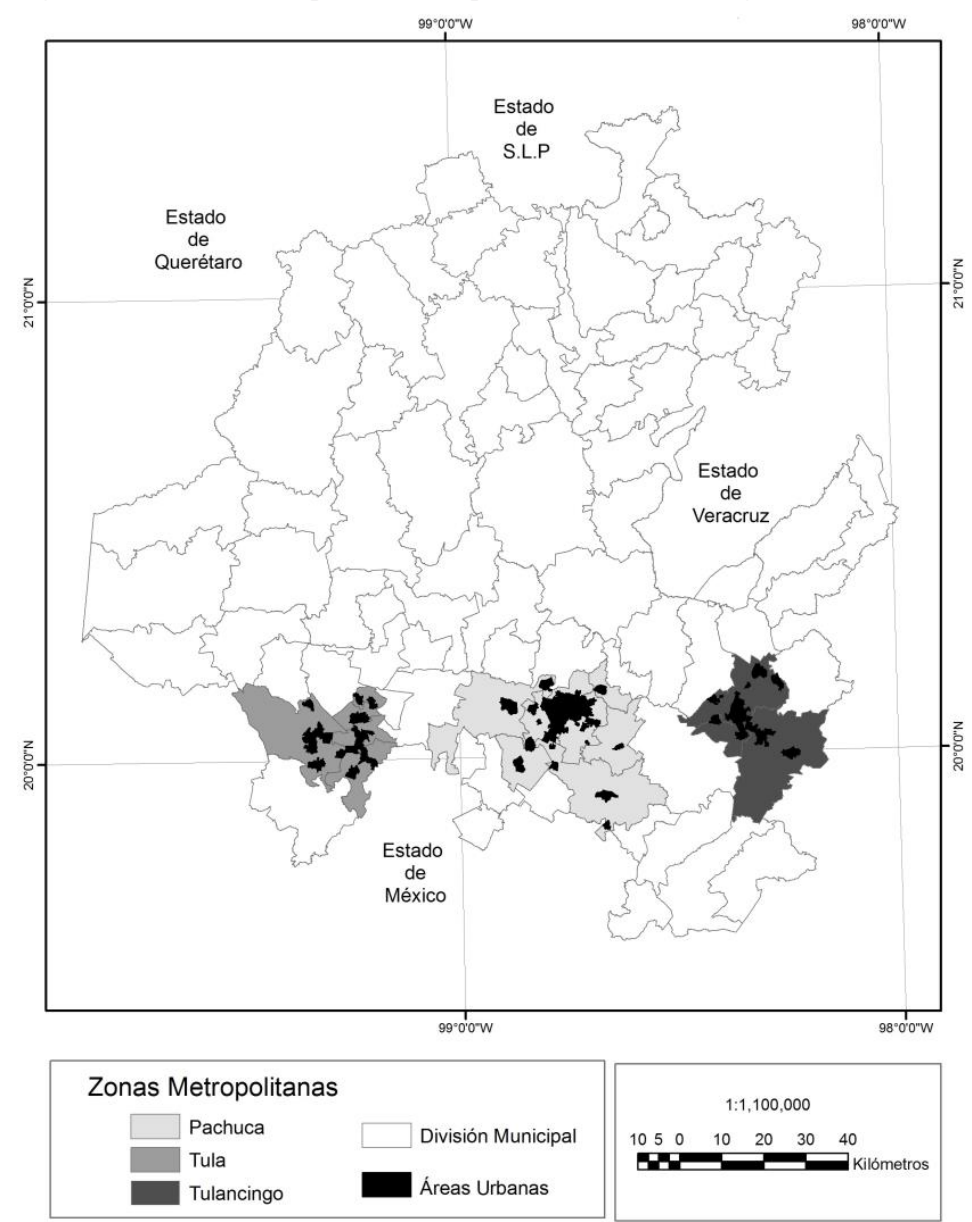

Fuente: Elaboración propia.

\section{ABordaje METOdológico}

Para referirnos a movilidad cotidiana reiteramos dos elementos básicos i) que es un desplazamiento entre unidades administrativas, con cierta regularidad o frecuencia, esto puede ser diario, varios días, o incluso una semana o más, caracterizándose como movilidad cotidiana al hecho de que siempre está presente el retorno al lugar de residencia; ii) la literatura presenta a esta movilidad cotidiana también referida como commute, la cual sería definida como viajes con cierta regularidad entre dos lugares como un suburbio y la ciudad (Jiménez, 2008). 
Una vez que se han planteado los elementos conceptuales acerca de la movilidad es conveniente presentar la estructura metodológica que seguirá el trabajo, y cómo se pretende llegar a la caracterización del perfil sociodemográfico de los commuters en las zonas metropolitanas del estado de Hidalgo. Primero se presenta un análisis descriptivo general de las variables que se pretenden modelar, seguido de la construcción y operacionalización de las variables; tanto independientes como dependiente, para cerrar con los resultados de una regresión logística binaria.

De esta forma, llegamos a los elementos a considerar como categoría de estudio y la variable dependiente. El commuting (categoría de estudio), se puede definir como el desplazamiento que se produce por la disociación entre lugar de trabajo y/o estudio y lugar de residencia. También, es preciso mencionar que a la persona que realiza este tipo de movimiento suele denominarse commuter (variable dependiente) (García, 2010).

\section{ANÁLISIS DESCRIPTIVO DE LA POBLACIÓN DE ESTUdIO}

Comencemos por dar un panorama general del comportamiento de la población objetivo, para esto haremos referencia al Tabla 1. Iniciemos por la variable dependiente, donde tenemos 107 mil 787 commuters, esta será nuestra población objetivo. Este dato lo comparamos respecto de la población total de las 
H (1 millón 038 mil 974 habitantes), mismo que parecería poco relevante, pero al segmentarlo y ver que representa el 25.6 por ciento la Población Económicamente Activa (PEA), da otra perspectiva, ya que esto significa que uno de cada cuatro habitantes de las ZMH que trabaja tiene que salir de su municipio de residencia. Así vemos que la población objeto de estudio tiene fuerte presencia y su estudio es de relevancia.

Continuando con el análisis de estos commuters que trabajan en un municipio diferente al que residen, la gran mayoría se mueve dentro de su ZM's. Al ser Hidalgo un estado encajado en el centro del país en el cual sus ZM's entran dentro de la categoría de ciudades medias. ${ }^{8}$ Los flujos son en varios sentidos, la conectividad, mercado de trabajo y servicios especializados hace que de las ZM's de Hidalgo salga población a trabajar de forma cotidiana.

El 65 por ciento de los commuters son hombres y 35 por ciento mujeres. Poco más del 65 por ciento de ellos tiene entre 30 y 59 años; aun cuando se reconoce en la literatura los roles marcados y definidos de la mujer que la apega a su lugar de residencia, vemos que en gran medida cuando vamos desglosando los datos el comportamiento de la mujer en el commuting no da muestra de diferencias abismales respecto a los hombres.

Cerca de 20 por ciento de los commuters no vivían en ese municipio cinco años antes, es decir son migrantes recientes y en su mayoría hombres. Además, poco más de 33 por ciento de las mujeres que son commuters en las ZM's de Hidalgo también son migrantes recientes. Esto es muestra de la participación de las mujeres en la economía familiar; su amplia incorporación al mercado laboral no les impide asumir los costos económicos o familiares de la movilidad cotidiana y están dispuestas a viajar a municipios fuera de donde residen. El cuestionamiento sería si las distancias recorridas son las mismas entre hombres y mujeres, situación que está fuera del objetivo de este trabajo.

En lo que concierne a escolaridad ${ }^{9}$ poco más de 45 por ciento de los hombres commuters tiene una baja escolaridad. De los commuters con

8 La ZM's de Tula tiene menos de 250 mil habitantes, que es el límite más utilizado en la literatura e instituciones oficiales para clasificar a las ciudades medias, aun cuando diversos documentos de instituciones como INEGI señalan como ciudades medias a las que tienen más de 100 mil habitantes. En este sentido, las tres ZM's de Hidalgo cumplen con elementos para ser considerarlas ciudades medias.

9 La escolaridad baja contempla a quienes solo tienen primaria y secundaria, la escolaridad media a las personas con nivel de bachillerato, la escolaridad alta a quienes tienen 
escolaridad media los porcentajes son muy similares entre hombres y mujeres. Porcentualmente las mujeres con escolaridad muy alta superan a los hombres en casi dos puntos porcentuales. Estos datos conducen a pensar que el perfil que concentra a gran cantidad de commuter está diferenciado claramente entre hombres con escolaridad media baja y que la mujer commuter presenta perfil con escolaridad más elevada.

Analicemos dos aspectos presentes en el Tabla 1. De los commuter hombres más del 62 por ciento son jefes de hogar, mientras que de las commuter mujeres solo el 28 por ciento son jefes de hogar. Esto ligado

licenciatura y la escolaridad muy alta a quien tiene un posgrado.

Tabla 1: Variables determinantes en el estudio del commuting

\section{COMMUTERS}

Commuters en las ZM's de Hidalgo * CONDICIONMIGRATORIA

Commuters que son migrantes recientes

EDAD

Población de commuters de menores de 15 años en las ZM's de Hidalgo *

Población de commuters 15 a 29 años en las ZM's de Hidalgo * Población de commuters 30 a 59 años en las ZM's de Hidalgo *

Población de commuters 60 y más años en las ZM's de Hidalgo*

ESCOLARIDAD

Commuters con escolaridad baja en las ZM's de Hidalgo*

Commuters con escolaridad media en las ZM's de Hidalgo*

Commuters con escolaridad alta en las ZM's de Hidalgo*

Commuters con escolaridad muy alta en las ZM's de Hidalgo*

JEFATURA DEL HOGAR

Jefes de hogar en las ZM's de Hidalgo*

No jefes de hogar en las ZM's de Hidalgo*

SITUACIÓN EN EL TRABAJO

Commuters con trabajo asalariado

Commuters que son patrones o empleadores

Commuters que son trabajadores por cuenta propia

Commuters que son trabajadores sin pago o que no

especificaron

\section{SITUACIÓN CONYUGAL}

Commuters unidos

Commuters solteros

Commuters alguna vez unidos

$\begin{array}{rrr}\text { HOMBRES } & \text { MUJERES } & \\ 69860 & 37927 & 107787 \\ & & \\ 13554 & 6816 & 20370\end{array}$

HOMBRES MUJERES

\begin{tabular}{|c|c|c|}
\hline $0.41 \%$ & $0.51 \%$ & \\
\hline $28.46 \%$ & $30.13 \%$ & \\
\hline $66.67 \%$ & $66.64 \%$ & \\
\hline $4.46 \%$ & $2.71 \%$ & \\
\hline 69860 & 37927 & 107787 \\
\hline $45.93 \%$ & $30.50 \%$ & \\
\hline $25.50 \%$ & $26.99 \%$ & \\
\hline $23.97 \%$ & $36.01 \%$ & \\
\hline $4.60 \%$ & $6.50 \%$ & \\
\hline 69860 & 37927 & 107787 \\
\hline $62.04 \%$ & $28.46 \%$ & \\
\hline $37.96 \%$ & $71.54 \%$ & \\
\hline 69860 & 37927 & 107787 \\
\hline $84.62 \%$ & $85.06 \%$ & \\
\hline $2.75 \%$ & $1.37 \%$ & \\
\hline $10.58 \%$ & $8.38 \%$ & \\
\hline $2.04 \%$ & $5.19 \%$ & \\
\hline 69860 & 37927 & 107787 \\
\hline $74.58 \%$ & $47.69 \%$ & \\
\hline $20.80 \%$ & $33.16 \%$ & \\
\hline $4.62 \%$ & $19.15 \%$ & \\
\hline 69860 & 37927 & 107787 \\
\hline
\end{tabular}

Fuente: Elaboración propia con datos de la Encuesta Intercensal 2015.

*En el estado hay 230329 commuters, las ZM'S concentran el 46.8 por ciento.

En las tres ZM'S hay 420906

PEA ocupadas, el 25.6 por ciento son commuters. 
a la situación conyugal de acuerdo al Tabla 1, señala que estos jefes de hogar son también personas unidas a una pareja, tanto en el caso de hombres como de mujeres. Entonces, el pensar en asumir ciertas distancias hasta un empleo ubicado en un municipio fuera del lugar de residencia queda relacionado al ser jefe de hogar sin importar el sexo. En el caso de los solteros, quizá no se asuma un traslado cotidiano y se opte por un cambio de residencia, en este sentido la mujer soltera commuter en proporción tiene mayor presencia, en el Tabla 1 observamos que los commuter solteros mujeres son poco más del 33 por ciento mientras que los hombres son poco más del 20 por ciento. Los commuters son en su mayoría asalariados (85 por ciento del total).

De esta forma, con la descripción general de estos datos, podemos decir que en mayor proporción el perfil de nuestro commuter es hombre entre 30 y 59 años, con escolaridad de baja a media, jefe de hogar, asalariado, unido y con gran posibilidad de ser migrante reciente. Y en menor proporción el perfil de nuestra commuter mujer entre 30 y 59 años con escolaridad media a alta, no jefe de hogar, asalariada y unida.

\section{CONSTRUCCIÓN DE LAS VARIABLES A UTILIZAR EN EL MODELO}

En México las fuentes que proporcionan información de calidad para conocer los desplazamientos al trabajo que presenta la población son el XII Censo General de Población y Vivienda 2000, II Conteo de Población y Vivienda 2005, Censo de Población y Vivienda 2010 y la Encuesta Intercensal 2015. ${ }^{10}$ En estas fuentes la variable lugar de trabajo está presente, pero no como tal una variable que se pueda definir como commuter, así que se tienen que construir recodificando una variable que nos ayude a diferenciar la población commuter. ${ }^{11}$

10 Los censos constituyen un procedimiento de generación de estadísticas que permite recabar información en todas y cada una de las unidades de observación de la población objeto de estudio. En este sentido ofrecen la posibilidad de obtener información con una cobertura total y amplios niveles geográficos de detalle: municipio, localidad, área geoestadística básica (AGEB) y manzana; además, existe la garantía de la calidad y la precisión de los resultados obtenidos. El Conteo de Población y Vivienda es un proyecto nacional que se realiza a la mitad del periodo del levantamiento de los Censos Generales de Población y Vivienda que se llevan a cabo cada diez años. La Encuesta Intercensal 2015 se llevó a cabo con la finalidad de actualizar la información sociodemográfica a la mitad del periodo comprendido entre el Censo de 2010 y el que habrá de realizarse en 2020. Aborda temas presentes en los últimos censos y guarda comparabilidad con ellos.

11 Primero necesitamos formular el lugar de residencia que aun cuando la encuesta tiene la variable "clave de la entidad" y "la clave del municipio" donde tiene su residencia habitual no están unidos en una misma variable así que se recodifica de forma tal que se unan la entidad y el municipio (Se trabajó con la base de datos de Hidalgo, de la Encuesta Intercensal 2015, así que la entidad en todos los casos es 13, y en el municipio tenemos la variable clave del municipio. Entonces la nueva variable lugar de residencia, quedaría 13 mil 048 que significa que la persona reside en Hidalgo en el municipio de 
Tabla 2: Recategorización de las variables a utilizar

\begin{tabular}{|c|c|c|c|}
\hline VARIABLE & TIPO DE VARIABLE & $\begin{array}{l}\text { PREGUNTA PLANTEADA Y } \\
\text { CATEGORIZACION }\end{array}$ & \\
\hline \multirow[t]{3}{*}{$\begin{array}{l}\text { Movilidad } \\
\text { cotidiana }\end{array}$} & Teórica dependiente & $\begin{array}{l}\text { ¿Trabaja en un municipio diferente a } \\
\text { su municipio de residencia? }\end{array}$ & \\
\hline & & Commuter & $1^{*}$ \\
\hline & & No commuter & 0 \\
\hline \multirow{3}{*}{ Sexo } & & Sexo & \\
\hline & Sociodemográfica independiente & Hombre & $1^{*}$ \\
\hline & & Mujer & 3 \\
\hline \multirow[b]{2}{*}{ Edad } & & ¿Cuántos años cumplidos tiene? & \\
\hline & Sociodemográfica independiente & $\begin{array}{l}\text { Se presenta la edad en años } \\
\text { desplegados** }\end{array}$ & \\
\hline \multirow{3}{*}{$\begin{array}{l}\text { Condición } \\
\text { migratoria }\end{array}$} & & $\begin{array}{l}\text { ¿Hace } 5 \text { años, en marzo del 2010, vivía } \\
\text { en el mismo municipio? }\end{array}$ & \\
\hline & Sociodemográfica independiente & Migrante & $1^{*}$ \\
\hline & & No migrante & 0 \\
\hline \multirow[b]{2}{*}{ Escolaridad } & & ¿Qué nivel de escolaridad tiene? & \\
\hline & Contextuales independientes & $\begin{array}{l}\text { Se presenta la escolaridad en años } \\
\text { desplegados** }\end{array}$ & \\
\hline \multirow[b]{3}{*}{ Jefatura del hogar } & & ¿Es usted el jefe del hogar? & \\
\hline & & Jefe & $1^{*}$ \\
\hline & Contextuales independientes & No jefe & 0 \\
\hline \multirow[b]{4}{*}{$\begin{array}{l}\text { Situación en el } \\
\text { trabajo }\end{array}$} & & ¿Cuál es su posición en el trabajo? & \\
\hline & & Trabajador asalariado & $1^{*}$ \\
\hline & & Patrón o empleador & 2 \\
\hline & Contextuales independientes & Trabajador por cuenta propia & 3 \\
\hline \multirow{4}{*}{$\begin{array}{l}\text { Situación } \\
\text { conyugal }\end{array}$} & & ¿Cuál es su situación conyugal? & \\
\hline & & Unido & $1^{*}$ \\
\hline & Contextuales independientes & Soltero & 2 \\
\hline & & Alguna vez unido & 3 \\
\hline \multirow[t]{3}{*}{$\begin{array}{l}\text { Propiedad de la } \\
\text { vivienda }\end{array}$} & Contextuales independientes & ¿Aquí vive el dueño de la vivienda? & \\
\hline & & Vive ahí el dueño & $1^{*}$ \\
\hline & & No vive ahí el dueño & 0 \\
\hline \multirow[t]{3}{*}{$\begin{array}{l}\text { Adquisición de la } \\
\text { vivienda }\end{array}$} & Contextuales independientes & ¿Cómo adquirió su vivienda? & \\
\hline & & Financiamiento por prestación laboral & $1^{*}$ \\
\hline & & Otro financiamiento & 0 \\
\hline
\end{tabular}

* Categorías de referencia.

** La variable edad y escolaridad se utiliza en años desplegados, así que la referencia es 0 años. Fuente: Elaboración propia con datos de la Encuesta Intercensal 2015.

Pachuca de Soto y cambiaría la terminación de acuerdo con el municipio al que se refiera). En una segunda instancia se necesita construir la variable que nos indique el lugar de trabajo (El lugar de trabajo lo construimos de la misma forma que el lugar de residencia), una vez que está en un mismo formato las dos variables "lugar de residencia" y "lugar de trabajo", lo que hacemos es una diferenciación, de quien reside y trabaja en 
Al momento de definir las variables independientes e ir a la base observamos que no necesariamente la categorización que se presenta de forma inicial es la más adecuada para ser explicada dentro del modelo, y en algunos casos es necesario la recodificación de nuevas variables utilizando elementos ya existentes. Las variables independientes a describir en esta sección se refieren a características sociodemográficas $^{12} \mathrm{y}$ del mercado laboral. El modo en que se reconfiguraron para esta investigación se presenta en el Tabla 2, donde se observan también las variables de referencia.

De inicio la primera variable que se relaciona a la movilidad cotidiana es la condición migratoria, nos referimos a la hipótesis que establece que la persona que en años recientes ha migrado, tiene mayor proporción a asumir la movilidad cotidiana al trabajo. De esta forma lo que deseamos conocer es el hecho de ser o no ser migrante reciente, ${ }^{13}$ con la mesura que en el análisis se deja fuera cualquier cambio de residencia hecho dentro de este intervalo de tiempo. Así esta variable es dicotómica, se diferencia en "Migrante" y "No migrante".

El nivel educativo se considera un determinante en la movilidad cotidiana al trabajo, generalmente también vinculado al ingreso, a mayor educación se puede suponer una mayor tendencia a la movilidad cotidiana por trabajo. Cabe mencionar que en el apartado descriptivo la mujer con alta escolaridad y entre los 15 y 29 años tiene mayor porcentaje en cuanto a movilidad cotidiana al trabajo. Para el nivel educativo se utilizó la variable escolaridad acumulada, que es continua y se da en años acumulados de escolaridad, se parte de la idea que a cada unidad más de escolaridad la probabilidad de ser commuter aumenta, por lo tanto se utiliza la variable desplegada.

El contar con casa propia y/o posibilidad de adquirirla a través de un crédito derivado de su trabajo, puede ser un aliciente para asumir todas las implicaciones de la movilidad cotidiana al trabajo. La operacionalización de estas variables se da como "vive ahí el dueño"

el mismo municipio y quien trabaja en un municipio distinto a donde reside. Así queda construida la variable dicotómica commuter, la cual será tomada como nuestra variable independiente, que a través de un modelo de regresión logística binaria se modelará el comportamiento de la movilidad cotidiana explicándolo mediante variables sociodemográficas y laborales, que se describen en el apartado siguiente.

12 La edad y el sexo se consideran las variables demográficas de partida para todo análisis por excelencia, dentro de esta sección se vinculará la edad y el sexo inmersa en el comportamiento de las demás variables.

13 Dentro de la Encuesta Intercensal 2015, conocemos el lugar de residencia cinco años antes, con ello se busca hacer dicotómica a la población que ha cambiado su residencia en este lapso. 
o "no vive ahí el dueño", además de "financiamiento por prestación laboral" u "otro financiamiento", así pretendemos relacionar a la movilidad laboral con la propiedad de la vivienda y el modo de adquisición de esta.

La condición de jefe de hogar vinculada a la movilidad cotidiana al trabajo deja de manifiesto que cuando se busca mejorar el nivel de vida de la familia, es el jefe quien asume los traslados más largos, siempre con la finalidad de que la familia cuente con mejores servicios de salud, educación, comerciales y otros beneficios que brindan ciertos espacios urbanos. Cuando el lugar de trabajo queda lejos del lugar de residencia la posibilidad de ser jefe de familia y migrar es más alta, en este caso no importaría si fuera hombre o mujer. La variable queda identificada como "jefe" o "no jefe".

Las variables independientes descritas hasta el momento pueden entrelazarse, si hablamos de jefe de hogar, podemos hablar de la situación conyugal, claro aquí podemos tener una gama amplia de categorías, la recategorización nos lleva solo a preguntar por los "unidos", "solteros" o "alguna vez unidos" para redefinir la variable, con la idea principal de que las personas unidas al ya tener un hogar familiar definido tienen más posibilidad de tener movilidad que los solteros a los que no les sería problemático decidir cambiar su residencia y es por una mejora en la rentabilidad de sus ingresos.

Por último, la posición en el trabajo se incluirá al modelado del commuting, se comenta que migra quien tienen mayor recurso, la hipótesis en este sentido sería, quien tiene una posición más privilegiada en el trabajo asume el commuting.

Todo lo anterior por supuesto afectado por la estructura etaria y el sexo, así los jóvenes en edades más productivas serían más propensos al commuting, en general por ser mayoría en la PEA pensaríamos que son los hombres los que dominan como commuters.

Se han descrito como se replantearon las variables a utilizar para la regresión logística de la movilidad cotidiana, planteando algunas ideas principales de cómo afecta cada variable a nuestro fenómeno de estudio, en este punto están listas para iniciar el procesamiento de

$$
\operatorname{Logit}(p)=\log \frac{P}{1-p}=\beta_{0}+\beta_{1} X_{1}+\beta_{2} X_{2}+\cdots \beta_{m} X_{m}
$$


datos, pero antes se presenta de forma general los elementos que se consideraron para la aplicación de un modelo de regresión logística binaria.

\section{GENERALIDADES DE LA REGRESIÓN LOGÍSTICA BINARIA}

Se plantea utilizar un modelo de regresión logística binaria, lo que pretende el modelo es cuantificar la dependencia o asociación entre una variable resultado (dependiente) y una o varias variables predictoras (independientes). Este modelo no presenta restricciones acerca de la naturaleza de las variables predictoras, pero sí se tienen restricciones para la variable resultado. La regresión logística binaria es el método de análisis adecuado cuando se necesita modelizar una variable respuesta binaria, y permite el uso conjunto de covariables de tipo categórico y continuo. (Hosmer y Lemeshow, 1989; SánchezCantalejo y Ramírez, 2000).

Consideremos la variable independiente que tiene que ser dicotómica como Y, además de un conjunto de variables predictoras $\mathrm{X}_{1}, \mathrm{X}_{2}, \ldots \mathrm{X}_{\mathrm{m}}$, tomadas de las características de $N$ individuos. El modelo logístico binario establece que:

Donde los $\beta_{\mathrm{i}}$ son los parámetros desconocidos del modelo.

Los resultados que arroja la regresión logística se interpretan a través de los Odds Ratio (OR) o razón de momios (RM). El Odds ratio (OR) que es un concepto ampliamente utilizado en la investigación social, el OR corresponde a la razón entre la probabilidad de que un evento ocurra y la probabilidad de que no ocurra, se ha traducido de múltiples formas al español: razón de oportunidades, razón de posibilidades, oportunidad relativa, razón de probabilidades o razón de productos cruzados (Aedo et al. 2010).

Un Odds Ratio es una división entre dos Odds, carece de unidades de medida y la Odds del grupo de interés se debe colocar siempre en el numerador y la de referencia en el denominador. Para poder interpretar una OR es necesario tener siempre en cuenta cuál es el factor o variable predictora que se estudia y cuál es el resultado o desenlace.

El valor nulo para la OR es el 1, una OR igual a 1 implica que las dos categorías comparadas son iguales en el caso de una OR menor a uno se reduce la probabilidad; cuando se tiene una OR mayor a 1 la probabilidad aumenta. El valor mínimo posible es 0 y el máximo teóricamente posible es infinito. Una OR inferior a la unidad se 
interpreta como que el desenlace es menos frecuente en la categoría o grupo que se ha elegido como de interés si en el grupo de referencia el evento ocurriera por azar.

Una vez que hemos dado un panorama general de lo que es y persigue una regresión logística, planteemos las variables a utilizar. Cabe mencionar que algunas de ellas no son factibles utilizarlas como están planteadas en nuestra base, así que necesitan crearse o recodificarse según sea el caso.

\section{El COMMUTING EN LA ZONAS METROPOLITANAS DE HidAlgo: UN ANÁLISIS APLICANDO REGRESIÓN LOGÍSTICA}

Para poder llegar a definir una caracterización general de las personas que presentan movilidad cotidiana al trabajo, que es lo planteado al inicio del artículo, se optó de acuerdo a elementos presentados en el apartado anterior, por la aplicación de una regresión logística binaria, ${ }^{14}$ con el fin de contrastar a la variable dicotómica commuter con una serie de variables explicativas relacionadas con características de índole socio demográfico y laboral. De acuerdo con Granados y Franco (2017), la información censal brinda información de ciertas características individuales que están ampliamente documentadas como determinantes en la movilidad laboral; mediante la utilización de los micro datos de la Encuesta Intercensal 2015, la cual contiene las variables explicativas a utilizar tales como edad, sexo, situación conyugal, lugar de residencia actual y cinco años atrás, lugar de trabajo, posición en el trabajo, jefatura familiar y escolaridad. De esta forma, se llega a la caracterización del perfil del commuter de las ZM's de Hidalgo.

La primera variable por considerar es la migración reciente. El Tabla 3 indica que el ser migrante reciente, ${ }^{15}$ aumenta 1.56 veces la posibilidad de ser commuter dentro de las ZM's de Hidalgo, esta idea contrastada con estudios similares como el de Granados y Franco (2017), para la zona centro de México o el de Ajenjo y Sabater (2004) en Cataluña, nos arroja diferencias. ${ }^{16} \mathrm{Al}$ analizar la Zona Centro de México en conjunto Granados y Franco señalan que el ser migrante disminuye

14 La regresión logística binaria es utilizada en casos que presentan una variable dependiente dicotómica, en este caso la variable es categorizada como commuter " 1 " y no commuter " 0 ".

15 Persona que en marzo del 2010 vivía en un municipio diferente a donde reside actualmente.

16 En el caso del estudio citado la diferencia esencial estaría explicada por el hecho de que la variable dependiente engloba a los commuters que son además migrantes recientes, 
un 37 por ciento la probabilidad de moverse cotidianamente por motivos de trabajo y reitera que la movilidad por motivos de trabajo no es realizada por migrantes recientes, sino por personas residentes del lugar. ${ }^{17}$ Aun cuando los porcentajes de commuters que además son migrantes recientes respaldan esta idea, la conjetura que se plantea en este artículo es contraria, reafirmando a la migración reciente como determinante de la movilidad cotidiana al trabajo, ya que autores como Pinto da Cunha señalan:

En la medida que el crecimiento metropolitano se debe en parte a la migración intrametropolitana, la movilidad cotidiana representa de alguna forma el resultado de esta migración (Cunha, 2006).

Por otra parte Ajenjo y Sabater (2004) en Cataluña señalan que el hecho de ser migrante reciente tiene un efecto positivo en la propensión a desplazarse a trabajar a municipios diferentes al de residencia, de hecho van un poco más allá al señalar que, ser un migrante reciente no solamente es sinónimo de mayor propensión a desplazarse para trabajar, sino que además, para los que se desplazan, también está relacionado con una mayor distancia entre la residencia y el lugar de trabajo. Además señalan que esto indicaría que la movilidad residencial no acerca a las personas a su lugar de trabajo, sino que en cierta medida las aleja, y por tanto sería necesario encontrar motivos ajenos al lugar de trabajo para los cambios residenciales. Los resultados de Ajenjo y Sabater (2004) son más acordes a lo encontrado en este artículo, aun cuando no incluimos información de distancias al trabajo, en ambos casos utilizamos a la migración reciente como variable explicativa, a diferencia de Granados y Franco (2017) que lo utilizan como parte de su variable a analizar.

Al respecto de la migración reciente la conclusión sería que i) es un determinante de la movilidad cotidiana al trabajo o commuting, ii) al analizar en su conjunto todo el centro de México y por otra parte solo las ZM's de Hidalgo llegamos a resultados distintos, por lo que al hablar de movilidad cotidiana y su relación con la migración reciente la dimensión de análisis juega un papel importante donde la intensión sería analizar en conjunto, pero también de forma separada

mientras que el estudio presentado en este artículo el ser migrante es una variable explicativa.

17 En este sentido los residentes del lugar serían los que cinco años antes ya vivían en ese lugar, aquí queda todavía la cuestión de la poca profundidad de los datos ya que deja fuera datos de cambios de residencia anteriores. 
las ZM's para observar las diferencias, tomando en cuenta que por la naturaleza de la información que tenemos no podemos contemplar los cambios de residencia hechos en periodos más cortos a cinco años, iii) el tener menor tiempo como residente de un lugar sería propenso a viajar distancias más largas, y iv) el haber migrado recientemente incrementa 56 por ciento la posibilidad de ser commuter.

El sexo y la edad como elementos fundamentales de cualquier análisis demográfico nos dice que el ser hombre dentro de las ZM's de Hidalgo aumenta 29.5 por ciento la probabilidad de ser commuter (ver Tabla 3), al respecto Concha y Pino (2017) señalan que en regiones de Chile como la recién decretada región de Ñuble el perfil del commuter es masculino predominantemente, lo mismo señala Granados y Franco (2017). Al analizar la región centro de México indica que el ser hombre aumenta 49 por ciento la probabilidad de tener desplazamientos por motivos de trabajo, atribuyendo esto a los roles que diferencian la actividad y por ende la movilidad de hombres y mujeres. En el análisis que realizan Ajenjo y Sabater (2004) en Cataluña, España, señalan

Tabla 3: Factores que inciden en la movilidad cotidiana al trabajo en las Zonas Metropolitanas de Hidalgo, 2015

\begin{tabular}{lcrrrrr}
\hline \multicolumn{1}{c}{ VARIABLES } & B & $\begin{array}{c}\text { Error } \\
\text { estándar }\end{array}$ & Wald & $\begin{array}{c}\text { Grados } \\
\text { de } \\
\text { libertad }\end{array}$ & Significancia & Exp(B) \\
\hline $\begin{array}{l}\text { No migrante } \\
\text { Soltero }\end{array}$ & 0.940 & 0.027 & 1251383 & 1 & .000 & 2.560 \\
$\begin{array}{l}\text { Alguna vez unido } \\
\text { Patrón o }\end{array}$ & 0.080 & 0.031 & 6722 & 1 & .010 & 1.083 \\
empleador & 1.074 & 0.036 & 0848 & 1 & .357 & 1.033 \\
$\begin{array}{l}\text { Trabajador por } \\
\text { cuenta propia }\end{array}$ & 0.181 & 0.061 & 8913 & 1 & .003 & 1.198 \\
$\begin{array}{l}\text { No jefe } \\
\text { Escolaridad }\end{array}$ & 0.096 & 0.021 & 21406 & 1 & .000 & 1.101 \\
$\begin{array}{l}\text { acomulante } \\
\text { Mujer }\end{array}$ & 0.028 & 0.001 & 500651 & 1 & .000 & 1.028 \\
$\begin{array}{l}\text { Edad } \\
\text { Constante }\end{array}$ & 0.259 & 0.020 & 167040 & 1 & .000 & 1.295 \\
\hline
\end{tabular}

Fuente: Elaboración propia con datos de la Encuesta Intercensal 2015.

Nota: Los resultados se leen respecto a la variable de referencia, ejemplo, el ser "soltero" aumenta 8.3 por ciento la probabilidad de ser commuter respecto del hecho de estar "unido".

la importancia de las características geográficas en el análisis de la movilidad cotidiana al trabajo. Refieren que para ciudades medias 
la importancia del sexo desaparece, no dándole un gran peso a la diferencia del sexo del commuter. Referente a la edad en los casos de los autores anteriores resaltan el impacto negativo de la edad donde por cada año de vida más del individuo su probabilidad de conmutar disminuye en poco menos del uno por ciento, cuestión similar a lo que podemos observar en la Tabla 3 donde la probabilidad de conmutar se reduce en 0.03 por ciento a cada año más de vida. En este artículo se usó la edad desplegada en años, es evidente que el impacto de la edad es muy bajo, cabe aclarar que se toma en cuenta a la PEA ocupada, que por definición está considerando a personas desde los 15 años que tenían empleo, ya que es una condiciónante para diferenciar a los commuters de los no commuter. Otra situación a resaltar sería que al ser larga la vida productiva de una persona, después de cierto número de años su propensión a ser commuter sería considerable, además que aun cuando el impacto de la edad en el hecho de ser commuter es bajo, su nivel de significancia hace resaltar el impacto de esta variable en la movilidad cotidiana.

La situación conyugal se planteó como variable explicativa para el commuting, donde el hecho de estar unido incrementa 8.3 por ciento la probabilidad de ser commuter. Autores como Ares (2010), señalan que la situación conyugal es un factor incidente en la movilidad cotidiana, ahonda en los tipos de hogar donde quien tiene una estructura de hogar donde existe pareja o hijos presenta mayor movilidad sobre quienes tienen estructura unipersonal. Esta condición coincide con lo que arroja el modelo que se presenta en este artículo, ya que el ser soltero no presentó significancia. Por otra parte, Granados y Franco (2017) refieren que los adultos jóvenes están más dispuestos a aceptar trabajos que requieren una elevada movilidad al principio de su carrera laboral que los trabajadores de mayor edad que ellos, ya que los jóvenes tienen mayor probabilidad de no tener pareja, no tener vivienda en propiedad, ni haber formado aun una familia los que expresan mayor disposición para la movilidad geográfica. Las aseveraciones a las que podemos llegar en este punto es que: i) el commuter es primordialmente una persona unida, con cierto arraigo en el lugar de residencia que lo lleva a la decisión de movilizarse al trabajo mientras la familia se queda en casa, ii) mientras que el soltero puede decidir un cambio de residencia de forma menos compleja, ya que no hay motivo alguno que le impida la movilidad, sumado a la factibilidad de conectividad que no le impedirá visitar a familia o 
amigos, y por otra parte, el poder rentar espacios a costos accesibles en el lugar donde se encuentre su trabajo, al ser una sola persona, iii) el hecho de ser soltero incrementa 8.3 por ciento la probabilidad de ser commuter, aun cuando la situación conyugal no tiene un grado de significancia.

Ligado a la situación conyugal está la jefatura familiar, partiendo de la idea que es el jefe de familia quien accede en determinado momento a la movilidad cotidiana esperando abastecer a la familia de un mayor nivel de vida generalizado, aun cuando se acepta los costos de la movilidad. Así vemos en la Tabla 3 que para las ZM's de Hidalgo el ser jefe de familia incrementa en un 10.1 por ciento la probabilidad de conmutar. En este punto vemos que Granados y Franco (2017) señalan que para la región centro de México el ser jefe de familia disminuye la probabilidad de ser commuter, esta situación no indica resultados distintos, la diferencia encontrada es que aquí se plantea a la población commuter y en el estudio referido se plantea la población commuter que además es migrante reciente, por lo que consideramos que los resultados presentan una lógica similar.

En lo concerniente a la posición del individuo en el trabajo, tenemos dos puntos a resaltar dentro de la Tabla 3, por una parte ser patrón o empleador eleva cerca de 193 por ciento la probabilidad de ser commuter, mientras que ser trabajador por cuenta propia eleva en un 19.8 por ciento la probabilidad de conmutar, esto nos indica que el perfil del commuter es en su mayoría personas con trabajo asalariado, además que quien es patrón o empleador asume también costos del commuting. En los estudios de commutig es una constante encontrar que la población que tiene mayor movilidad se encuentra ocupada en sectores asalariados primordialmente relacionados a servicios, porque las condiciones de seguridad que brindan son más atractivos, así como por la idea de ter un empleo seguro bien remunerado que permita tener acceso a prestaciones y que asegure un incremento del bienestar familiar, es la idea que acerca a la población a los empleos asalariados (Granados y Franco, 2017; Ares, 2010; Ajenjo y Sabater, 2004; Concha y Pino, 2017).

La escolaridad juega un papel primordial en la decisión de buscar empleo fuera del lugar de residencia, la Tabla 3 resalta que a cada año más de escolaridad que presente una persona se incrementa en 2.8 porciento la probabilidad de trabajar en un municipio distinto al de residencia. El nivel de escolaridad está relacionado a la posición en 
el trabajo, ya que ésta demanda cierto perfil educativo que limitaría en cierto sentido la movilidad (Granados y Franco, 2017; Ares, 2010; Ajenjo y Sabater, 2004). Al respecto podemos decir que, i) el nivel educativo del commuter está relacionado al tipo de empleo, entonces si el empleo es de bajo perfil la escolaridad del commuter será baja, ii) el commuter entre mayor escolaridad tiene mayor es la posibilidad de aceptar la movilidad, ya que se asume que el empleo al que accedería tendría mejores condiciones que le permitirían asumir mayores costos.

Cerrando este apartado demos un panorama del perfil del commuter de las ZM's de Hidalgo para el 2015. Encontramos que la participación de la mujer es fuerte, sin embargo, predominantemente el commuter es hombre, unido, jefe de familia, con trabajo asalariado donde la escolaridad demandada es media-baja y, el commuter a medida que tiene más edad es menos propenso a conmutar, además con alta probabilidad de ser migrante reciente. La comparación empírica con otros estudios similares indican las variables más representativas en el fenómeno del commuting es la condición migratoria, la posición en el trabajo, la condición de jefe de familia; el ser hombre o mujer, la edad, la educación o la situación conyugal, aun cuando son variables representativas, la literatura no les da el suficiente peso en la decisión de la movilidad cotidiana al trabajo.

\section{Conclusiones}

La movilidad cotidiana es inminente en regiones como la del centro de México, las ZMH no están exentas de la movilidad cotidiana dentro de la región, su conectividad hace factible los desplazamientos cotidianos. $\mathrm{Al}$ analizar elementos sociodemográficos como la condición migrante, la edad, el sexo, la escolaridad, la jefatura de hogar, la situación conyugal y la posición en el trabajo, a través del análisis descriptivo y la modelación mediante una regresión logística simple, se observa que la principal concomitante del commuting es la condición migrante, es decir, las personas que commuters de las ZMH son personas que migraron en años recientes, esto refuerza el planteamiento teórico que presenta la idea de la toma de decisión entre migrar a una ciudad que brinde mejor nivel de vida aun cuando se asuman los costos de un traslado cotidiano al trabajo. Este argumento daría el indicio que quienes pueden asumir estos costos de traslado son personas que cuentan con una mejor posición en su trabajo, en este sentido el modelo indica que ser patrón o empleador aumenta casi el 20 por 
ciento la posibilidad de migrar, esto sin dejar de lado que el commuter por excelencia es trabajador asalariado.

Existen elementos como la situación conyugal o la jefatura del hogar que parecen no ser de gran impacto, sin embargo, son considerados determinantes ya que el apego a la familia determina esa disposición a asumir los costos del commuting, y esta situación la presentan las personas unidas y que son jefas de hogar, sin importar si son hombres o mujeres.

Aun cuando en la parte de análisis descriptivo, la educación hace una clara diferenciación que ubica a la mujer con alta escolaridad como quien más posibilidad tiene de ser commuting, el modelo nos dice que cada año de escolaridad acumulada aumenta 2.8 por ciento la posibilidad de ser commuter. La única variable que hace disminuir la probabilidad de ser commuter es la edad, ya que a cada año más de edad la posibilidad de ser commuter disminuye un 0.03 por ciento.

En conclusión, por una parte las personas jóvenes con mayor educación son más propensas a ser commuters, además, las personas unidas o solteras no presentan grandes diferencias al momento de asumir estos traslados cotidianos al trabajo y que una persona que ya ha migrado recientemente asume más fácilmente estos viajes cotidianos al trabajo.

\section{REFERENCIAS BIBLIOGRÁFICAS}

Aedo, Sócrates, Pavlov Stefanía, Clavero Francisca. (2010). "Riesgo relativo y Odds ratio ¿Qué son y cómo se interpretan?”, Rev. Obstet. Ginecol. - Hosp. Santiago Oriente Dr. Luis Tisné Brousse, vol. 5 (1), pp. 51-54. Disponible en ttps://www.researchgate.net/publication/251573463_Riesgo_relativo_y_Odds_ratio_Que_son_y_como_se_interpretan

Ajenjo, M. y Sabater, A. (2004). "El impacto de los movimientos migratorios sobre la movilidad habitual por trabajo en Cataluña", Geo Crítica / Scripta Nova. Revista electrónica de geografía y ciencias sociales. Barcelona: Universidad de Barcelona, 1 de febrero, Vol. VIII(158). Disponible en http://www.ub.es/geocrit/sn/sn-158.htm (ISSN: 1138-9788)

Ares, Sofía Estela. (2010). "Espacio de vida y movilidad territorial habitual en Chapadmalal, Buenos Aires, Argentina Cuadernos de Geografía", Revista Colombiana de Geografía, No. 19, pp. 27-40 Universidad Nacional de Colombia Bogotá, Colombia. Disponible en http://www.redalyc.org/ articulo.oa?id=281822029003 
Boix Rafael y Trullén Joan. (2012). Policentrismo y estructuración del espacio: una revisión: crítica desde la perspectiva de los programas de investigación; ACE. VI(18). Disponible en https://upcommons.upc.edu/bitstream/ handle/2099/11680/ACE_18_SE_20.pdf

Casado, J. M. (2008). "Estudios sobre movilidad cotidiana en México". Scripta Nova. Revista Electrónica de Geografía y Ciencias sociales. Barcelona: Universidad de Barcelona, 15 de septiembre, XII(273). Disponible en http://www.ub.es/geocrit/sn/sn-273.htm

Concha Murua, Gary y Pino Arriagada, Osvaldo. (2017). "Características de los conmutantes en Chile: una mirada desde la creación de regiones", Revista Academia \& Negocios, 3(1) pp. 51-68, ISSN: 0719-7713 / 07196245, Universidad de Concepción. Disponible en http://ran.udec.cl/ojs/ index.php/ran/article/view/43/83

Cunha, José Marcos Pinto da. (2006). "Dinâmica migratória e o processo de ocupação do Centro-Oeste brasileiro: o caso de Mato Grosso", Revista Brasileira de Estudos de População, Campinas, vol. 23, junio.

García Cruz, Domingo Rafael. (2010). Movilidad Intermetropolitana en la región del Centro de México. El patrón del commuting en el México Central en el 2000; Ponencia presentada en la X Reunión Nacional de Investigación Demográfica en México, "Escenarios Demográficos y Política de Población en el Siglo XXI", Sociedad Mexicana de Demografía, en El Colegio de México, Ciudad de México, el 5 de noviembre.

Granados, J. A. y Franco, L. M. (2017). “Migración y movilidad laboral entre las zonas metropolitanas de la región centro de México", Papeles de población CIAEP/UAEM, No. 91, pp. 117-141. Disponible en http://rppoblacion. uaemex.mx/pp/index.php/papelesdepoblacion/article/view/779/pdf

Hosmer, y S. Lemeshow. (1989). Applied Logistic regression. Wiley series in probality and statistics, 2 ed.

Jiménez, Maren Andrea. (2008). "Los Censos de 2010 y la migración interna, internacional y otras formas de movilidad territorial", Potencialidades de la Medición de la Movilidad Cotidiana a Través de Los Censos Seminario-Taller, Santiago de Chile 10 al 12 de diciembre.

Instituto Nacional de Estadística y Geografía (INEGI). (2015). Encuesta Intercensal 2015: síntesis metodológica y conceptual.

Organización Internacional para las Migraciones (OIM). (2015). Los migrantes y las ciudades: Nuevas colaboraciones para gestionar la movilidad, Informe Sobre Las Migraciones 2015, Suiza.

Recio, Albert; Banyuls, Josep; Cano, Ernest; y Miguélez, Fausto. (2006). "Migraciones y mercado laboral", Revista de Economía Mundial, No. 14, pp. 171-193 Sociedad de Economía Mundial Huelva, España, disponible en http://www.redalyc.org/articulo.oa?id=86617194007

Roncayolo, Marcelo. (1988). La Ciudad, Siglo XXI, España. 
Sánchez, Cantalejo y Ramírez, Sergio. (2000). Regresión logística en salud Publica, Escuela Andaluza de salud pública, Granada España.

Susino, Joaquín y Martínez Reséndiz, Juana. (2009). Movilidad cotidiana por trabajo en el análisis de la estructura territorial: Andalucía y Centro de México, Universidad de Granada/Universidad Nacional Autónoma de México.

Villanueva, Omar. (2013) (n.p.). Dinámica de la población en tres polígonos de la Zona Metropolitna de Pachuca. Maestría. Universidad Autónoma del Estado de Hidalgo.

\section{RESUMEN CURRICULAR DE LOS AUTORES}

\section{Omar Villanueva Mejía}

Ingeniero industrial por la Universidad Autónoma del Estado de Hidalgo. Maestro en Estudios de Población por la Universidad Autónoma del Estado de Hidalgo. Estudiante del Doctorado en Estudios de Población de la Universidad Autónoma del Estado de Hidalgo. Líneas de investigación: Migración interna y movilidad cotidiana. Dirección electrónica: villanueva_mejia_omar@hotmail.com

\section{Laura Myriam Franco Sánchez}

Licenciada en economía por la Universidad Autónoma del Estado de Hidalgo. Maestra en Estudios de Regionales por el Instituto Dr. José María Luis Mora, Doctora en Urbanismo por la Universidad Nacional Autónoma de México. Coordinadora de la licenciatura en desarrollo regional de la Universidad Autónoma del Estado de Hidalgo. Profesora investigadora de tiempo completo del área académica de sociología y demografía de la Universidad Autónoma del Estado de Hidalgo miembro del Sistema Nacional De Investigadores Nivel 1. Líneas de investigación: Cambio Social ante Procesos de Desarrollo, procesos migratorios y mercados de trabajo.

Dirección electrónica: lfranco@uaeh.edu.mx

Sergio Cuauhtémoc Gaxiola Robles Linares

Actuario por la Facultad de Ciencias de la Universidad Nacional Autónoma de México. Maestro en Estudios de Población por la Facultad Latinoamericana de Ciencias Sociales (sede México). Doctor en Estudios de Población por El Colegio de México. Profesor de Tiempo Completo del Centro de Investigación y Estudios Avanzados de la Población de la Universidad Autónoma del Estado de México (UAEM). 
Docente en programas de licenciatura de la UAEM. Ha publicado varios artículos. Líneas de investigación: envejecimiento, mercados de trabajo y pobreza.

Dirección electrónica: serobles99@gmail.com 